\title{
Determining the ultrasound time of response using FPGA-based electronics read-out
}

\begin{abstract}
This paper presents a system to measure accurate value of time of response (TOR) for ultrasound transducer pairs based on Field Programmable Gate Arrays (FPGA). In the experiment, the transmitter is set to transmit the pulse to the target object and the reflected signal is then captured by the receiver. The time taken for the signal to travel back to the receiver is measured by TOR. FPGA technology and data acquisition system were used to generate a pulse at the transmitter site. The pulse is projected directly to the targeted object and the signal that has been reflected from the object will be captured three times by the receiver. The results show that the TOR value obtained can be calculated accurately. The best TOR found in this study is the signal capture 1 with the lowest percentage error of $0.819 \%$.
\end{abstract}

Keyword: FPGA; Sensors; TOR; Ultrasound transducer 